\title{
INSTANTANEOUS PARAMETER IDENTIFICATION FOR MILLING FORCE MODELS USING BAYESIAN OPTIMIZATION
}

\author{
B. Schmucker ${ }^{1 *}$, M. Busch ${ }^{1}$, T. Semm ${ }^{1}$, M. F. Zaeh ${ }^{1}$ \\ ${ }^{1}$ Technical University of Munich, Institute for Machine Tools and Industrial Management, Garching near Munich, \\ Germany \\ *Corresponding author; e-mail: benedikt.schmucker@iwb.tum.de
}

\begin{abstract}
The comparison between measured and simulated machining forces enables the evaluation of workpiece quality, process stability, and tool wear condition. To compute the machining forces that occur, mechanistic cutting force models are typically used. The cutting force coefficients (CFCs) of mechanistic force models are directly linked to the mechanics of chip formation and, thus, depend on the toolworkpiece combination and on the prevailing cutting conditions. CFCs are usually identified via the average cutting force identification method, which requires the execution of cutting tests under defined test conditions. Hence, determining CFCs for different cutting conditions is time-consuming and expensive. In this paper, the performance of an instantaneous CFC identification approach based on Bayesian Optimization during the machining of arbitrary workpiece geometries is studied. Bayesian Optimization is well suited for global optimization problems with computationally expensive cost functions. The simulated cutting forces are calculated using a dexel-based cutter workpiece engagement simulation and the actual cutting forces are measured during the machining process using a dynamometer. Thus, an efficient identification of CFCs could be achieved.
\end{abstract}

\section{Keywords:}

machining simulation; parameter identification; optimization; data analytics

\section{INTRODUCTION AND LITERATURE REVIEW}

Milling processes have been continuously improved over the past decades through enhancements in machine dynamics [Zaeh 2019], tool design [Stone 2014], and process control [Schmucker 2021]. Many of these advances have been achieved using so-called digital process twins [Haenel 2019] or virtual machining simulations [Altintas 2005], which, among other things, estimate the machining forces that occur. The calculation of the process forces supports, on the one hand, the selection of the process parameters and the tool path planning. On the other hand, the comparison between simulated and measured forces allows to monitor the workpiece quality [Scippa 2013; Denkena 2012], to evaluate the process stability [Aslan and Altintas 2018], and to determine the current tool wear condition [Altintas and Aslan 2017].

For computing milling forces, analytical, empirical, numerical, and mechanistic cutting force models are available. Mechanistic methods are of recent origin and are suitable for the use in machining processes with complex tool geometries and tool-workpiece engagement conditions. In addition, mechanistic models are able to consider effects of process noise such as runout [Wimmer 2018], tool wear, and material inhomogeneities [Ehmann 1997]. All these effects are covered by the so-called cutting force coefficients (CFCs), which must be calibrated based on measured machining forces. Usually, the calibration involves recording cutting forces under various defined cutting conditions (e.g., feed per tooth, cutting speeds), and then identifying the model parameters using optimization or curve fitting algorithms.

Cutting forces during machining can be measured via direct or indirect techniques. Direct methods are characterized by using piezoelectric dynamometers, spindle integrated force sensors, or strain gauges close to the cutting region. But they can also be estimated indirectly from motor current measurements, encoder data [Yamada 2017], or vibrations [Albertelli 2016]. Conventionally, the so-called average cutting force identification method is used to determine the CFCs for mechanistic cutting force models. However, this approach usually relies on additional cutting tests due to the predefined experimental conditions. Consequently, this offline approach is time-consuming and costly and does not provide any information about the evolution of the model parameters during machining operations [Farhadmanesh and Ahmadi 2020].

In order to detect the changes of the coefficients during machining or during the service life of a tool, two requirements arise for the identification method.

1. Online capability: The identification should be performed swiftly and parallel with machining operations. 
2. Flexible identification: No customized experimental setup should be necessary, but the manufacturing of an arbitrary workpiece geometry shall be used for the parameter identification.

CFCs of mechanistic force models are directly linked to the mechanics of chip formation and, thus, depend on the characteristics of the shearing action, friction on the rake face, and the ploughing action under the flank face [Farhadmanesh and Ahmadi 2020]. According to Gross [2015], the following three approaches are available to study these effects and, therefore, to calibrate CFCs:

1. Orthogonal Coefficient Identification

2. Average Cutting Force Identification

3. Instantaneous Cutting Force Identification

Orthogonal Coefficient Identification

In the orthogonal coefficient identification method, the CFCs are determined in turning tests and then transformed to oblique cutting conditions [Budak 1996]. Since this method requires not only special cutting conditions but also extra tools and workpieces, it can be denied online capability. Furthermore, orthogonal coefficients cannot be applied to milling operations with complex tool geometries (e.g., unequal pitch angles, serrated tools). Both requirements mentioned above - online capability and flexible identification - are therefore not fulfilled by this approach.

\section{Average Cutting Force Identification}

In the Average Cutting Force Identification (ACFI) method, CFCs are determined by performing linear regressions of the average cutting forces acquired at different feed rate per tooth settings. Hereby, the forces are averaged over one or more tool revolutions and, therefore, tool runout and instantaneous force modulations have no effect on the identified coefficients. In summary, it can be stated that this method is not suitable for the CFC identification when machining arbitrary geometries, and the introduced requirements are not satisfied here as well.

\section{Instantaneous Cutting Force Identification}

Among the above listed methods, only the instantaneous coefficient identification is suitable to detect the evolution of the force coefficients during the machining operation. In instantaneous approaches, the cutting constants are identified by fitting simulated and measured forces in the time domain [Grossi 2015]. In this case, the forces do not have to be measured at various feed per tooth settings but simulated and measured cutting forces must be synchronized in a first step. Various optimization approaches were already presented in the literature, which perform an instantaneous CFC determination after a successful synchronization [Wimmer 2018; Gonzalo 2010; Rivière-Lorphèvre 2017].

Grossi [2015] have employed a genetic algorithm for the instantaneous CFC identification. By combining this with the compensation of the dynamometer dynamics via a Kalman Filter, they were able to determine speed-varying cutting force constants in high-speed machining applications. In addition, Farhadmanesh and Ahmadi [2020] have introduced three different identification methods for instantaneous CFC calibration. The presented Recursive Least Squares, Kalman Filter, and Extended Kalman Filter algorithms are suitable for a rapid synchronization and a subsequent recursive determination of the cutting force coefficients.

All these approaches have in common that they rely on a prior synchronization of the cutting force simulation and measurement, and that they cannot handle varying cutter workpiece engagements (CWEs) in the identification data sets.

Due to this, a novel identification method based on Bayesian Optimization is presented in this publication (see Section 3.2). This optimization method enables an efficient search of global extrema even for computationally expensive cost functions. To avoid a synchronization of measured and simulated cutting forces, the so-called Dynamic Time Warping is used as the error function in the optimization procedure (see Section 3.1). In addition, instead of a classical analytical cutting force model, a geometric, tri-dexel based CWE simulation (see Section 2) with subsequent conversion to cutting forces is used to allow an identification for arbitrary workpiece geometries as well. Finally, the presented procedure is compared with experimental results and it is evaluated whether the defined requirements - online capability and flexible identification are met (see Section 4 and 5).

\section{PROCESS FORCE SIMULATION}

\subsection{Mechanistic Force Model}

There are several mechanistic force models available in literature. One of the most widely used is the mechanistic cutting force model by Budak [2006], which is characterized by good adaptability and proven accuracy. The process force simulation of this publication is also based on this mechanistic model. Therefore, this approach will be presented in the following.

The cutting force model describes for a point on the $j$ th cutting tooth three differential force components, which are the tangential force component $d F_{t j}$, the radial force component $d F_{r j}$, and the axial force component $d F_{a j}$ (see Figure 1). In linear mechanistic modelling, all these force components are assumed to be linear functions of the instantaneous chip thickness:

$$
\begin{aligned}
& d F_{t j}(\Phi, z)=\left[K_{t e}+K_{t c} h_{j}(\Phi, z)\right] d z, \\
& d F_{r j}(\Phi, z)=\left[K_{r e}+K_{r c} h_{j}(\Phi, z)\right] d z, \text { and } \\
& d F_{a j}(\Phi, z)=\left[K_{a e}+K_{a c} h_{j}(\Phi, z)\right] d z,
\end{aligned}
$$

where $K_{q c}$ and $K_{q e}(q \in\{t, r, a\})$ represent the cutting force and edge-force coefficients. In the context of this publication, only these six parameters are considered as CFCs. Runout parameters are neglected at this point.

The axial and radial depth of cut $a_{p}$ and $a_{e}$, the number of teeth $N$, the cutter diameter $D$, and the helix angle $\beta$ determine what portion of the tooth is in contact with the workpiece for a given angular orientation of the cutter $\Phi=\Omega t$, where $\Omega$ ist the angular speed $\Omega=2 \pi n / 60, t$ is the time, and $n$ is the spindle speed in revolutions per minute.

The immersion angle $\Phi$ of tooth $j$ is measured with reference to the feed direction, which is the $x$-direction in Figure 1. This difference compared to the model of Budak [2006] was introduced to simplify the integration of the dexel-based CWE simulation (see Section 2.2) into the coefficient identification. Thus, the chip thickness is calculated based on the feed per tooth $f_{z}$ and the instantaneous immersion angle $\Phi(z)$ at axial position $z$ :

$h_{j}(\Phi, z)=f_{z} \cos (\Phi(z))$

Assuming that the position of the first cutting edge is known, the immersion angle $\Phi(z)$ of the $N$ flutes can be calculated by

$$
\Phi_{j}(\Phi, z)=\Phi+(j-1) \frac{2 \pi}{N}-\frac{\tan (\beta)}{\frac{D}{2}} z .
$$


The engagement limits of the individual cutting edges at the various axial positions are defined as follows:

$\Phi_{s}(z) \leq \Phi_{j}(\Phi, z) \leq \Phi_{e}(z)$

The start and exit angles $\Phi_{s}$ and $\Phi_{e}$ are also dependent on the $z$-position and can also vary along the $z$-axis for more complex workpiece geometries.

After transforming the differential cutting forces into the global tool coordinate system ( $x-, y-$, and $z$-direction, see Equation 6), an integration along the tool axis is carried out and the forces of all $N$ teeth are summed up:

$$
\begin{aligned}
& F_{x}(\Phi)=\sum_{j=1}^{N} \int_{z_{1}}^{z_{2}} d F_{x, j}(z, \Phi), \\
& F_{y}(\Phi)=\sum_{j=1}^{N} \int_{z_{1}}^{z_{2}} d F_{y, j}(z, \Phi), \\
& F_{z}(\Phi)=\sum_{j=1}^{N} \int_{z_{1}}^{z_{2}} d F_{z, j}(z, \Phi) .
\end{aligned}
$$

For simple cutting conditions, the integration limits $z_{1}$ and $z_{2}$, and the entry and exit angles $\Phi_{s}$ and $\Phi_{e}$ can be easily determined to solve these equations [Wimmer 2018]. However, this is not possible with arbitrary workpiece geometries and with changing CWEs. Due to this, this publication relies on a dexel-based CWE simulation, which is described in the following section.

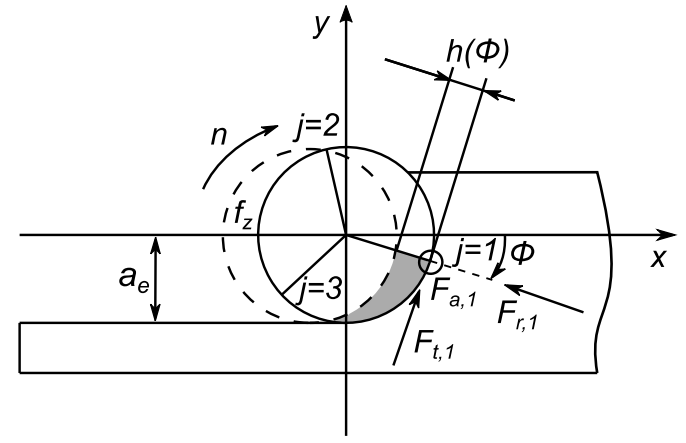

Figure 1: Geometry of an end-milling process

\subsection{Dexel-based Cutting Force Simulation}

CWE or material removal simulations have been in the focus of science for many years. These models operate by creating virtual representations of workpieces and removing any material that interferes with the cutter geometry of a tool, which is moved along a path [Armendia 2019]. Many of these methods are quite computationally expensive, but voxel ("volume elements") and dexel ("depth elements") models have proven to be practical even in realtime applications [Witt 2019]. The use of the ModuleWorks API (API: application programming interface) is particularly widespread in the area of machining simulation [Armendia 2019; Haenel 2019]. This $\mathrm{C} / \mathrm{C}++$ library is based on the socalled tri-dexel model, where a volume is approximated by parallel line grids in three perpendicular planes. This publication makes use of the ModuleWorks software module as well.

In the developed process force simulation, the toolpath is divided into individual cutter locations. The distance between these positions defines the accuracy of the CWE simulation and, thus, of the cutting force calculation. This spacing should not be greater than the feed per tooth $f_{z}$, so that the effects of the individual cutting edges can be studied, and the instantaneous force modulation can be computed.

At each discrete tool position, it is calculated which area of the cutter is currently engaged (see exemplary green CWE marking in Figure 2). In addition, the tool is discretized into individual elements in radial $(d \theta)$, and tool profile direction $(\beta)$. Furthermore, the elements are constrained in axial direction by the element height $\left(b_{e l}\right)$. Elements belonging to cutting edges are called cutting elements and contribute to the cutting force calculation. In contrast, the non-cutting elements are neglected in the simulation.

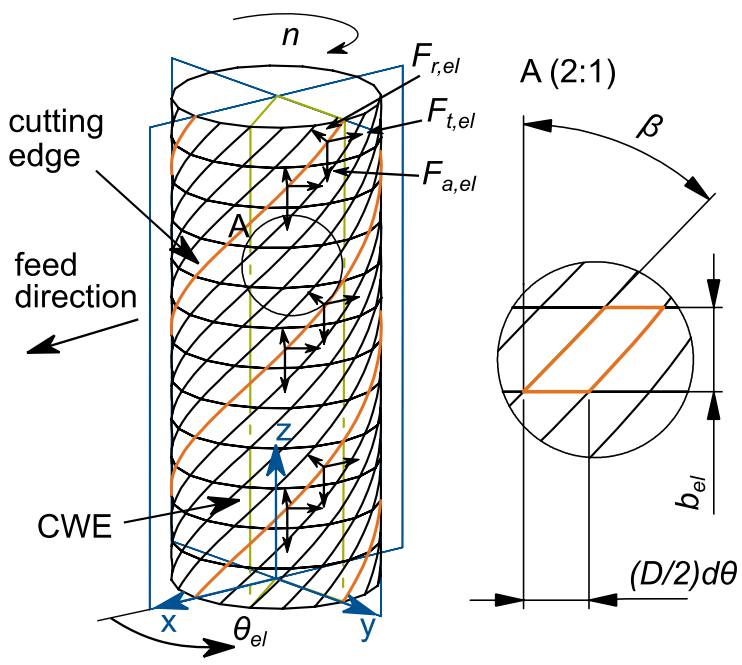

Figure 2: Tool discretization along the tool profile of a helical end-milling cutter

Figure 2 shows two different coordinate systems. The tool coordinate system with the axes $x, y$, and $z$ is aligned with the tool orientation. Additionally, the illustration depicts the local $\{r t a\}$ element coordinate system (radial, tangential, and axial axis), whose orientation depends on the position on the tool profile.

If a cutting element is engaged, its contribution to the cutting force is calculated as follows:

$\left\{\begin{array}{l}F_{r, e l} \\ F_{t, e l} \\ F_{a, e l}\end{array}\right\}=\left\{\begin{array}{l}K_{r c} \\ K_{t c} \\ K_{a c}\end{array}\right\} h_{e l} b_{e l}+\left\{\begin{array}{l}K_{r e} \\ K_{t e} \\ K_{a e}\end{array}\right\} b_{e l}$.

The depth of the element engagement $h_{e l}$ is calculated using equation 2 . In this case, the immersion angle $\Phi$ equals the angular position of the elements $\theta_{e l}$. Since the local discrete cutting forces of an element are determined in the $\{r t a\}$ coordinate system, a coordinate transformation must be performed:

$\left\{\begin{array}{l}F_{x, e l} \\ F_{y, e l} \\ F_{z, e l}\end{array}\right\}=\boldsymbol{T}\left\{\begin{array}{l}F_{r, e l} \\ F_{t, e l} \\ F_{a, e l}\end{array}\right\}, \boldsymbol{T}=\left\{\begin{array}{ccc}-\cos \left(\theta_{e l}\right) & -\sin \left(\theta_{e l}\right) & 0 \\ -\sin \left(\theta_{e l}\right) & \cos \left(\theta_{e l}\right) & 0 \\ 0 & 0 & -1\end{array}\right\}$.

After performing this transformation, all element forces share a common coordinate system, the $\{x y z\}$ tool coordinate system. Therefore, the proportions of the elements $N_{e}$ can be summed up numerically:

$\left\{\begin{array}{l}F_{x} \\ F_{y} \\ F_{z}\end{array}\right\}=\sum_{0}^{N_{e}-1}\left\{\begin{array}{l}F_{x, e l} \\ F_{y, e l} \\ F_{z, e l}\end{array}\right\}$.

Elements that are not assigned to a cutting edge or that are not engaged do not contribute to the calculation of the cutting force. The element forces of these elements have the value $F_{q, e l}=0 N(q \in x, y, z)$ in all three coordinate directions.

\subsection{Generic Process Forces as an Input Format for the Optimization}

The simulation approach presented in the previous section allows the estimation of cutting forces along arbitrary tool paths as a function of engagement ratios and CFCs. However, if the dexel-based simulation approach is to be used to calibrate these coefficients, an exchange format 
between simulation and optimization is needed. For this purpose, so-called generic process forces (GPFs) have been introduced:

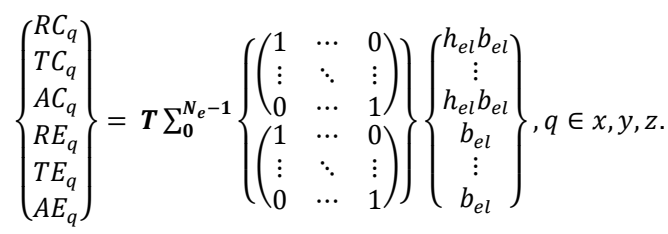

With the help of the following equations, the cutting forces can be reconstructed from the GPFs. Thus, these GPFs ( $R C$ / $R E$ : radial generic cutting / edge forces, $T C$ / $T E$ : tangential generic cutting / edge forces, $A C$ / $A E$ : axial generic cutting / edge forces) are suitable to avoid a repetitive execution of the simulation during the optimization.

$\left\{\begin{array}{l}F_{x} \\ F_{y} \\ F_{z}\end{array}\right\}=\left\{\begin{array}{lll}R C_{x} & T C_{x} & A C_{x} \\ R C_{y} & T C_{y} & A C_{y} \\ R C_{z} & T C_{z} & A C_{z}\end{array}\right\}\left\{\begin{array}{l}K_{r c} \\ K_{t c} \\ K_{a c}\end{array}\right\}+\left\{\begin{array}{lll}R E_{x} & T E_{x} & A E_{x} \\ R E_{y} & T E_{y} & A E_{y} \\ R E_{z} & T E_{z} & A E_{z}\end{array}\right\}\left\{\begin{array}{c}K_{r e} \\ K_{t e} \\ K_{a e}\end{array}\right\}$

Thus, the calculation of actual simulation forces based on the GPFs is suitable for use in the error function of the optimization described in the following section.

\section{IDENTIFICATION OF CUTTING FORCE COEFFICIENTS}

The aim of this publication is to present how the coefficients of the mechanistic cutting force model can be determined based on captured process forces and a geometric CWE simulation. In each iteration step, it is necessary to determine the error between simulated and measured forces. Usually, least squares methods are used for this purpose in the literature [Grossi 2015; Gonzalo 2010; Farhadmanesh and Ahmadi 2020]. However, in order to avoid a synchronization of measured and simulated data and to allow varying engagement conditions during coefficient estimation, Dynamic Time Warping (DTW) is used in this work as the error function (see Section 3.1). Since DTW is a computationally complex error function, a Bayesian Optimization approach is used for identifying the CFCs. This optimization technique is explained at the end of this chapter (see Section 3.2).

\subsection{Dynamic Time Warping}

DTW is a well-known method to find an optimal alignment between two given time series. In simple terms, the sequences are distorted - warped - in a nonlinear way to fit them to each other (see Figure 3 a). Originally, DTW was used for the comparison of different speech patterns in automatic speech recognition. But DTW has also been successfully used in areas such as data mining and information retrieval, to automatically deal with temporal deformations and different speeds associated with timedependent data [Müller 2007]. In addition, DTW was applied in the field of machining, namely in chatter detection [Yesilli 2019].

DTW is an algorithm which is able to measure the similarity between two time series even if they have dissimilar length: $\widehat{F}_{q}=\left\{\widehat{F}_{q, 1}, \widehat{F}_{q, 2}, \ldots, \hat{F}_{q, k}, \ldots, \hat{F}_{q, n}\right\}, n \in \mathbb{N}$ and

$F_{q}=\left\{F_{q, 1}, F_{q, 2}, \ldots, F_{q, l}, \ldots, F_{q, m}\right\}, m \in \mathbb{N}$.

The simulated $\left(\hat{F}_{q, k}\right)$ and the measured $\left(F_{q, l}\right)$ forces can be arranged to form an $n$-by- $m$ plane, where each grid point corresponds to an alignment between the elements $\hat{F}_{q, k}$ and $F_{q, l}$. A so-called warping path $W$ (see Figure $3 \mathrm{~b}$ ) aligns the elements so that the distance between the signals is minimized.
$W_{q}=\left\{W_{q, 1}, W_{q, 2}, \ldots, W_{q, w}, \ldots, W_{q, p}\right\}, w \in \mathbb{N}$

The length $p$ of the warping path fulfills the constraint $m \leq p \leq n$, if $m \leq n$ is assumed [Yesilli 2019].

Various metrics are available for evaluating the distance between two points. In the implementation associated to this publication, the Euclidean distance is used:

$d\left(\hat{F}_{q, k}, F_{q, l}\right)=\left\|\hat{F}_{q, k}-F_{q, l}\right\|_{2}=\sqrt{\left(\hat{F}_{q, k}-F_{q, l}\right)^{2}}, q \in x, y, z$

Once a distance measure is selected, DTW can be defined as a minimization problem over the warping paths:

$\operatorname{DTW}\left(\hat{F}_{q, k}, F_{q, l}\right)=\min \left(\sum_{w=1}^{L} d\left(w_{w}\right)\right), q \in x, y, z$.

The length of the warping path $L$ is determined by the ratio of $n$ and $m$. The distance measure DTW is calculated separately for the three force directions $x, y$, and $z$ and then averaged as follows:

$e_{D T W}=\frac{D T W\left(\hat{F}_{x, k}, F_{x, l}\right)+D T W\left(\hat{F}_{y, k}, F_{y, l}\right)+D T W\left(\hat{F}_{y, k}, F_{y, l}\right)}{3}$.

The calculation of the value $e_{D T W}$ (see equation 15) is applied as the error function in the Bayesian Optimization, which is explained in the following section.

a)

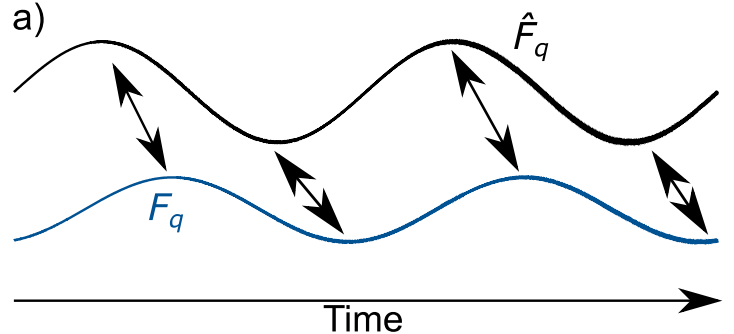

b)

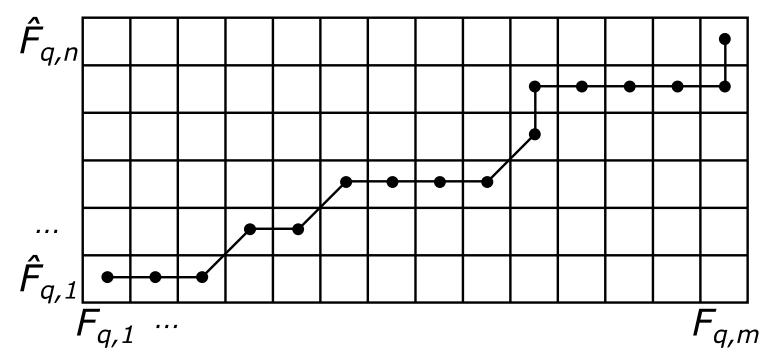

Figure 3 : Schematic drawing of Dynamic Time Warping based on Müller [2007]; a) warping / distortion of a time series; b) exemplary warping path

\subsection{Bayesian Optimization}

Bayesian Optimization (BO) is an optimization method that is well known from the field of Automated Machine Learning. In Automated Machine Learning it is used for the selection of so-called hyperparameters. Hyperparameters determine how well artificial intelligence algorithms can learn from the provided training data. For instance, the number of hidden layers, the number of neurons in each hidden layer, the activation function, and the learning rate of artificial neural networks are hyperparameters. The challenge in determining these hyperparameters is that the execution and the validation of the learning procedure are computationally intensive. Since the identification of CFCs in combination with DTW shows the same challenge, it is reasonable to apply $\mathrm{BO}$ here as well.

\section{Sequential Model-Based Optimization}

BO is a sequential model-based optimization approach mainly composed of a surrogate model and an acquisition function. The surrogate model is intended to represent objective functions, such as:

$\hat{x}=\arg \min f(x)$. 
The first step (see Table 1) is to draw an initial sample for $x$ (e.g., sample point $x_{1}$ in Figure 4) and to evaluate the error function for this choice.

Table 1 : Sequential Model-Based Optimization based on Archetti and Candelieri [2019]

\begin{tabular}{c|l}
\hline step & description \\
\hline 1 & initial sample of the surrogate model \\
\hline 2 & $\begin{array}{l}\text { acquisition function identifies new promising } \\
\text { points }\end{array}$ \\
\hline 3 & evaluation of the objective function \\
\hline 4 & update of the surrogate model \\
\hline 5 & $\begin{array}{c}\text { checking the termination criteria: } \\
\bullet \quad \text { if fulfilled go to step 6 } \\
\end{array}$ \\
\hline 6 & else go back to step 2 \\
\hline
\end{tabular}

Subsequently, it is the task of the acquisition function to select at which point the next comparison between the actual error function and the surrogate model should be performed (e.g., $x_{2}$ or $x_{3}$ in Figure 4).

Two different approaches, exploration and exploitation, can be used for this second step of the sequential optimization. Exploration means sampling at locations where the prediction uncertainty is high (see $A$ in Figure 4). On the contrary, exploitation describes the idea of sampling in areas with high fidelity (see $B$ ).

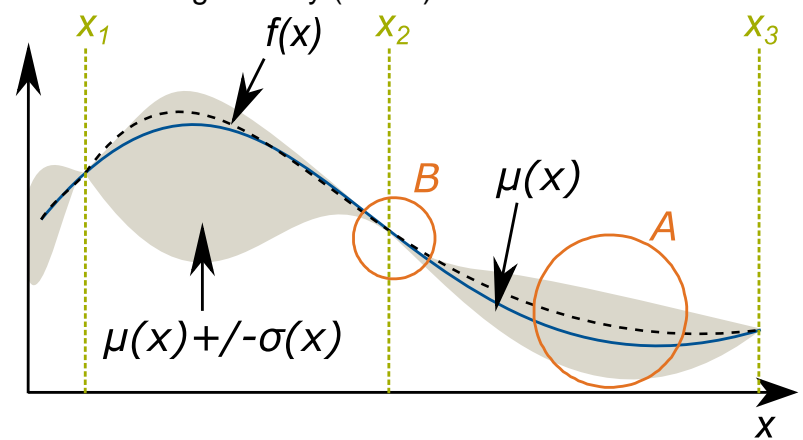

Figure 4: Schematic drawing of Bayesian Optimization using a probabilistic surrogate model based on Archetti and Candelieri [2019]: $f(x)$ : error function; $\mu(x)$ mean of the surrogate model; $\sigma(x)$ : standard deviation; A: exemplary area for exploration; $B$ : exemplary area for exploitation

After each selection of new samples, the distance to the real data (see Equation 15) is evaluated and the surrogate model is updated based on these observations (see steps 3 and 4). Thus, the approximation of the surrogate model to the actual error function becomes progressively closer and the uncertainty in the prediction decreases. This procedure is pursued until a chosen termination criterion is reached and the "best seen" solution is returned as the optimization result (see steps 5 and 6 ).

\section{Surrogate Model}

To enable the distinction between exploration and exploitation areas, the surrogate model must provide a prediction probability. Due to this, a probabilistic surrogate model, a multivariate Gaussian Process (GP), has been used in the implementation associated with this publication. A GP is specified by its mean function

$\mu(x)=\mathbb{E}[f(x)]$

and by the corresponding covariance function

$k\left(x, x^{\prime}\right)=\mathbb{E}\left[(f(x)-\mu(x))\left(f\left(x^{\prime}\right)-\mu\left(x^{\prime}\right)\right)\right]$.
The covariance function (see Equation 17 and 18) is also called kernel in the context of GPs. However, it must be mentioned that $\mathrm{BO}$ is not necessarily based on a GP as a surrogate model. Other probabilistic approaches or also deterministic functions are applicable [Archetti and Candelieri 2019].

\section{Acquisition Function}

The acquisition function determines at which point, $x$, the agreement of the surrogate model and the actual error function should be evaluated next. Hence, this type of function determines the distribution between exploration and exploitation. Various acquisition functions are available in the literature, starting from early implementations such as "Probability of Improvement" [Kushner 1964] but also more recently published approaches such as "K-Optimality" [Yan 2018]. In the identification of CFCs presented here, the "Expected Improvement" approach is used. This acquisition function evaluates the expected amount of improvement in the objective function, ignoring values that cause an increase in the prediction uncertainty [Archetti and Candelieri 2019].

In the following section, it is shown how this sequential optimization method can be used for the determination of the CFCs, and how this combination of a surrogate model and an acquisition function leads to a rapid and reliable coefficient identification.

\section{EXPERIMENTAL RESULTS}

\subsection{Test Setup and Design of Experiments}

To investigate the $\mathrm{BO}$ based CFC identification, the experimental setup depicted in Figure 5 was used. A Kistler dynamometer (type: 9257B) was mounted on the machine table of a milling center GROB G352T. During cutting tests with various process parameters (see Table 2), the spindle was solely moved in $x$-direction for feed.

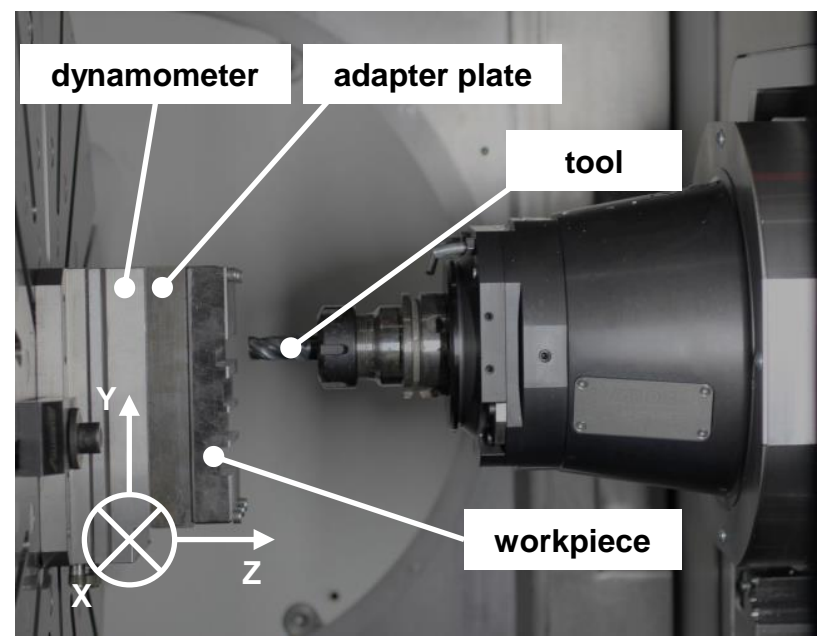

Figure 5 : Test setup for CFC identification

In order to provide a broad database for the evaluation of the coefficient identification approach, 23 machining tests were performed. On the one hand, the process parameters spindle speed $n$ and feed rate $v_{f}$ and, on the other hand, the CWE conditions were varied. The radial depth of cut $a_{e}$ was increased from $4 \mathrm{~mm}$ to $16 \mathrm{~mm}$ and the axial cutting depth $a_{p}$ from $3 \mathrm{~mm}$ to $5 \mathrm{~mm}$. This has resulted in quarter, half, three-quarter, and full immersion cuts and, thus, the cutting forces were acquired at a wide range of engagement ratios. 
Table 2: Test plan for the identification of the cutting force coefficients

\begin{tabular}{|c|c|c|c|c|}
\hline $\begin{array}{c}\text { test } \\
\text { no. }\end{array}$ & $\begin{array}{c}\boldsymbol{v}_{\boldsymbol{f}} \\
\text { in } \mathrm{mm} / \mathrm{min}\end{array}$ & $\begin{array}{c}\boldsymbol{n} \\
\text { in rev/min }\end{array}$ & $\begin{array}{c}\boldsymbol{a}_{\boldsymbol{p}} \\
\text { in } \mathrm{mm}\end{array}$ & $\begin{array}{c}\boldsymbol{a}_{\boldsymbol{e}} \\
\text { in } \mathrm{mm}\end{array}$ \\
\hline 1 & 500 & 2000 & 3 & 16 \\
\hline 2 & 350 & 2000 & 3 & 16 \\
\hline 3 & 650 & 2000 & 3 & 16 \\
\hline 4 & 750 & 3000 & 3 & 16 \\
\hline 5 & 400 & 1600 & 3 & 16 \\
\hline 6 & 500 & 2000 & 4 & 16 \\
\hline 7 & 500 & 2000 & 5 & 16 \\
\hline 8 & 500 & 2000 & 3 & 12 \\
\hline 9 & 350 & 2000 & 3 & 12 \\
\hline 10 & 650 & 2000 & 3 & 12 \\
\hline 11 & 750 & 3000 & 3 & 12 \\
\hline 12 & 400 & 1600 & 3 & 12 \\
\hline 13 & 500 & 2000 & 3 & 8 \\
\hline 14 & 350 & 2000 & 3 & 8 \\
\hline 15 & 650 & 2000 & 3 & 8 \\
\hline 16 & 750 & 3000 & 3 & 8 \\
\hline 17 & 400 & 1600 & 3 & 8 \\
\hline 18 & 500 & 2000 & 3 & 4 \\
\hline 19 & 350 & 2000 & 3 & 4 \\
\hline 20 & 650 & 2000 & 3 & 4 \\
\hline 21 & 750 & 3000 & 3 & 4 \\
\hline 22 & 400 & 1600 & 3 & 4 \\
\hline 23 & 500 & 2000 & 3 & 16 \\
\hline
\end{tabular}

The workpiece material and the tool were not modified during the experiment. The workpiece material was 1.1730 carbon tool steel $(\mathrm{C} 45 \mathrm{U})$, and a solid carbide three flute end-milling cutter (Miller OptiMill-Uni M3033) with a diameter of $16 \mathrm{~mm}$ and a helix angle of $30^{\circ}$ was used for the cutting experiments. The supply of cooling lubricant was switched off.

\subsection{Evaluation of the coefficient identification}

Figure 6 shows the measured and simulated cutting forces in $x$-, $y$-, and $z$-direction for test no. 1 . The simulation was performed according to the descriptions in Section 2 and on the basis of the identified CFCs using the BO approach (see Table 3).

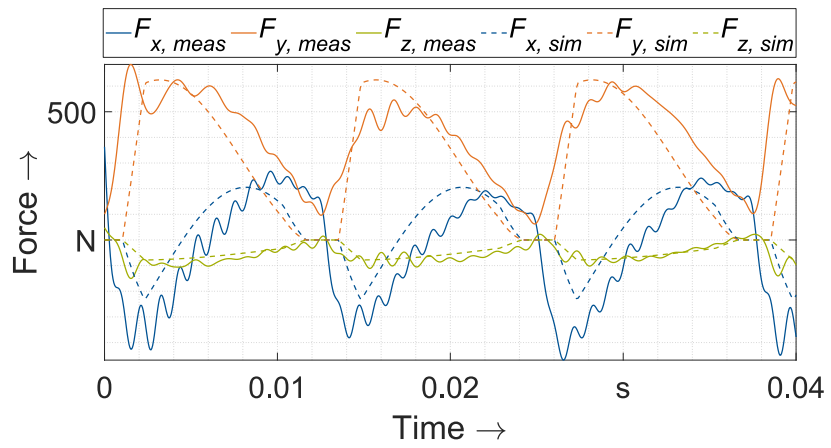

Figure 6 : Measured $\left(F_{x, \text { meas }}, F_{y, \text { meas }}, F_{z, \text { meas }}\right)$ and simulated cutting forces $\left(F_{x, \text { sim }}, F_{y, \text { sim }}, F_{z, \text { sim }}\right)$ in $x-, y$-, and $z$-direction for test no. 1

It can be clearly seen that there is a good agreement between the simulated and the measured values. In all three directions, both amplitude and shape are well approximated. The presented result is already achieved after a calculation time of $7.3 \mathrm{~s}$. The required optimization time to achieve comparable results was significantly higher when using Genetic (GA) or Particle Swarm (PSA) algorithms with $119.0 \mathrm{~s}$ and $72.9 \mathrm{~s}$, respectively.

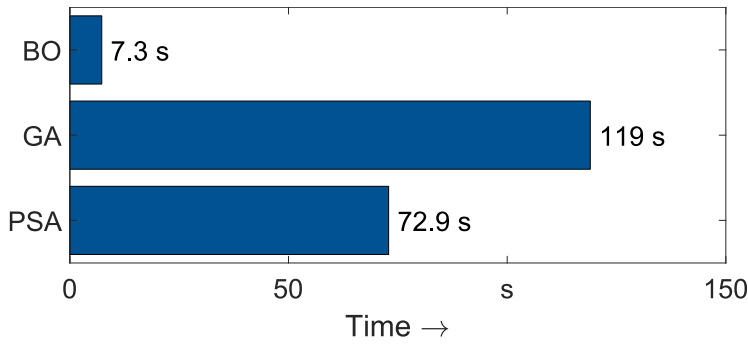

Figure 7: Required time for the identification run using different optimization methods for test no. 1; computing unit: Intel Core ${ }^{\mathrm{TM}}$ i7 $\mathrm{CPU}$ with $1.80 \mathrm{GHz}$

Additionally, a second instantaneous identification result is listed in Table 3. In this identification run, all 23 process parameter settings were considered instead of individual cutting conditions. At least one tool revolution from each test was incorporated into the identification data set. This identification setup can be compared to the acquisition of cutting forces on arbitrary workpiece geometries with changing CWEs. The simulation data were created according to the experimental conditions to provide generic process forces as the second data source for the optimization. The advantage of using DTW as error function is that no time-consuming and error-prone synchronization between the two time series, measured cutting forces and simulated generic process forces, has to be performed.

Table 3: Identified cutting force coefficients for two different optimization runs

\begin{tabular}{|c|c|c|c|c|c|c|}
\hline method & $\begin{array}{c}\boldsymbol{K}_{\boldsymbol{r c}} \\
\text { in } \\
\mathrm{N} / \mathrm{mm}^{2}\end{array}$ & $\begin{array}{c}\boldsymbol{K}_{\boldsymbol{r e}} \\
\text { in } \\
\mathrm{N} / \mathrm{mm}^{2}\end{array}$ & $\begin{array}{c}\boldsymbol{K}_{\boldsymbol{t c}} \\
\text { in } \\
\mathrm{N} / \mathrm{mm}^{2}\end{array}$ & $\begin{array}{c}\boldsymbol{K}_{\boldsymbol{t e}} \\
\text { in } \\
\mathrm{N} / \mathrm{mm}\end{array}$ & $\begin{array}{c}\boldsymbol{K}_{\boldsymbol{a c}} \\
\text { in } \\
\mathrm{N} / \mathrm{mm}\end{array}$ & $\begin{array}{c}\boldsymbol{K}_{\boldsymbol{a c}} \\
\text { in } \\
\mathrm{N} / \mathrm{mm}\end{array}$ \\
\hline BO & 1142.6 & 47.7 & 1500.5 & 56.7 & 311.0 & 9.4 \\
\hline PSA & 1200.0 & 55.5 & 1500.0 & 46.1 & 200.0 & 19.3 \\
\hline GA & 1188.7 & 46.8 & 1500.1 & 55.6 & 238.58 & 16.1 \\
\hline \hline \multicolumn{7}{|c|}{ test nos. 1-23 } \\
\hline BO & 1067.6 & 11.3 & 2018.5 & 27.7 & 209.7 & 8.7 \\
\hline PSA & 1058.0 & 11.1 & 2082.3 & 29.7 & 303.7 & 4.2 \\
\hline GA & 1027.9 & 13.2 & 2032.0 & 27.2 & 270.7 & 5.8 \\
\hline \hline ACFI & 1016.0 & 25.5 & 2108.0 & 27.0 & 409.2 & 1.5 \\
\hline
\end{tabular}

Even with this enlarged input data set, BO optimization can be performed much faster than the optimizations based on GA or PSA algorithms. The time required was only $46.1 \mathrm{~s}$ compared to $4560.1 \mathrm{~s}$ (GA) and $4287.8 \mathrm{~s}$ (PSA).

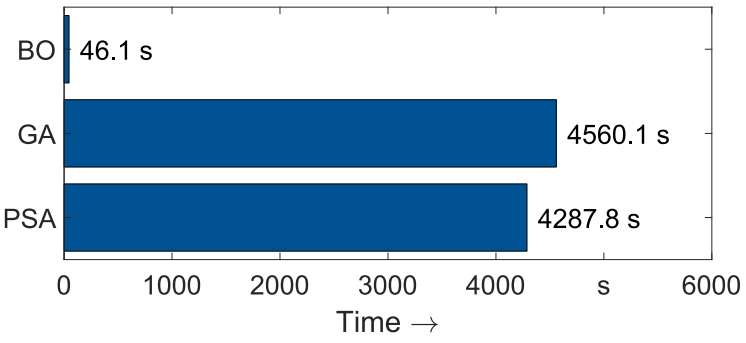

Figure 8: Required time for the identification run using different optimization methods for test nos. 1-23; computing unit: Intel Core ${ }^{\mathrm{TM}}$ i7 $\mathrm{CPU}$ with $1.80 \mathrm{GHz}$ 


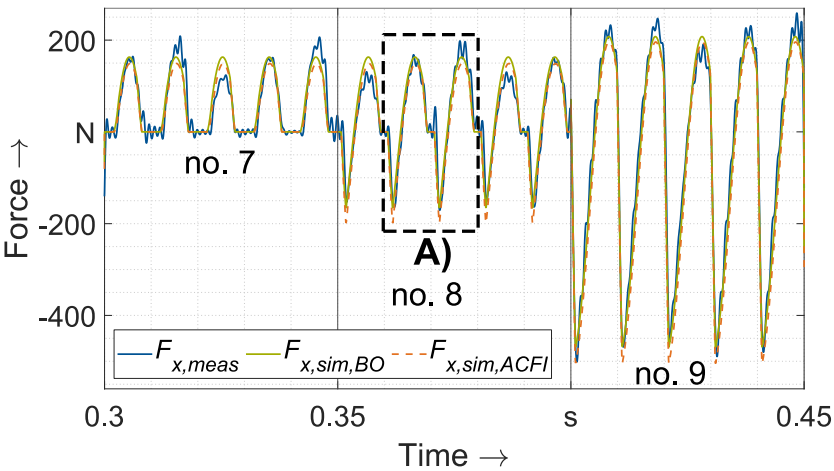

A)

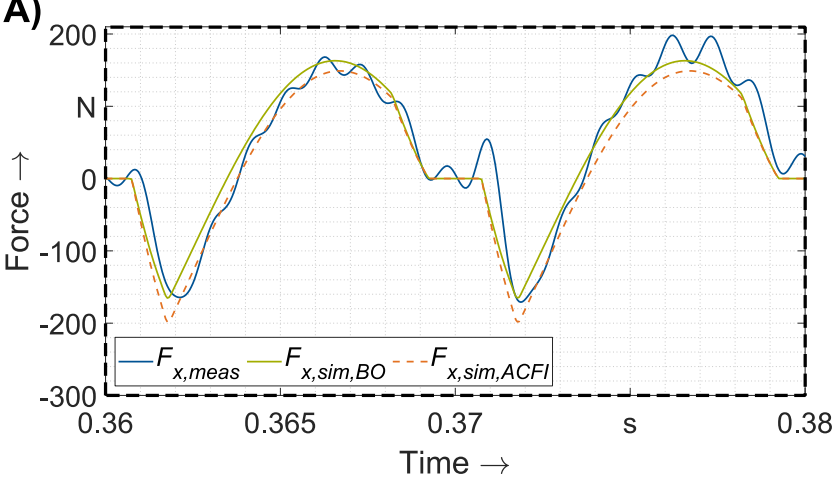

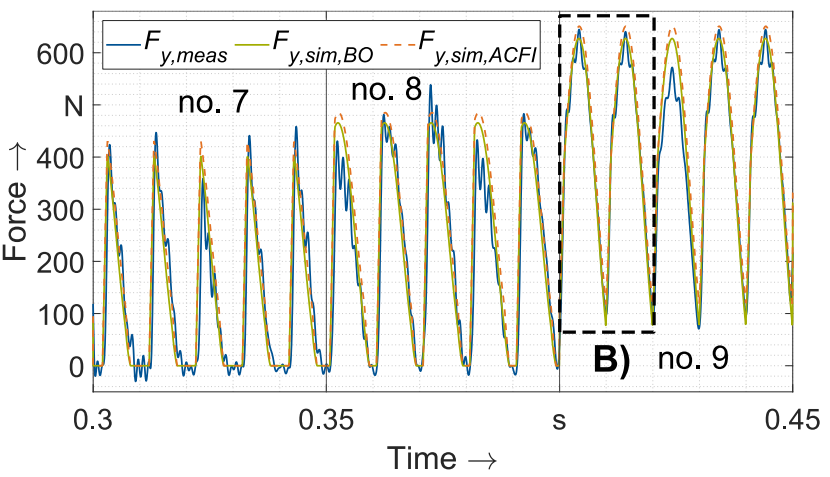

B)

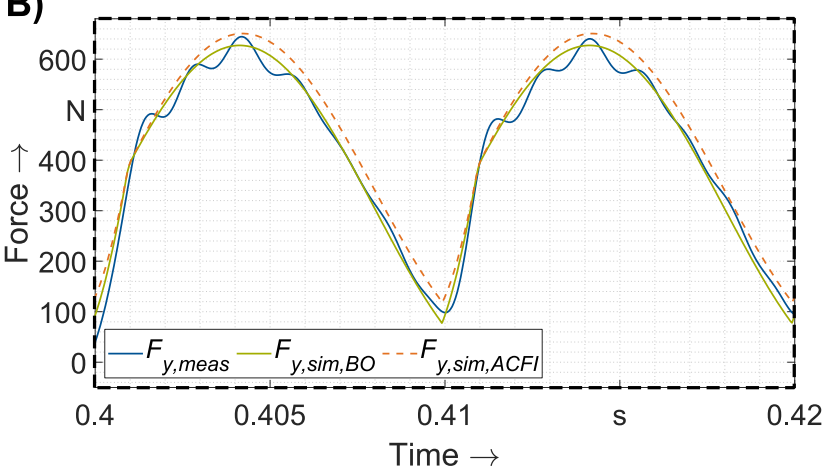

Figure 9 shows the low-pass filtered (cut-off frequency $=1 \mathrm{kHz}$ ) measured cutting forces and also the simulated values based on the coefficients identified via BO and ACFI. For better visibility, only the $x$ - and $y$-axis cutting forces of the machining tests 7 to 9 are shown. It can be noted that the agreement between simulated and measured forces is not inferior for the BO method compared to the widely used ACFI approach. Even to the contrary, the normalized root-mean-square error compared to the measured forces is reduced from $7.5 \%$ to $7.3 \%$ by using $\mathrm{BO}$. This is where the advantage of the introduced instantaneous CFC determination becomes apparent, as different CWE conditions can be considered during identification.

\section{CONCLUSION AND OUTLOOK}

Within this publication a novel identification method is presented to identify cutting force coefficients for mechanistic force models and to monitor their evolution during machining operations. Conventional CFC calibration methods, e.g., ACFI, require executing cutting tests under defined test conditions, and processing them with offline curve fitting algorithms. This is time-consuming and costly.

In the previous sections, it is shown that the combination of a dexel-based process force simulation (see Section 2) and an instantaneous identification approach based on BO (see Section 3) enables a rapid determination of the CFCs. For both, simple engagement conditions and more complex input data, this approach is significantly faster than other frequently used optimization methods, such as GA and PSA (see Figure 7 and Figure 8). The presented approach is therefore more suitable for online use in parallel with machining operations. As a result, tasks such as tool wear monitoring can be implemented more effectively and competitively with this approach.

However, the estimation of the calculation time (see Figure 7 and Figure 8) is subject to some uncertainties. On the one hand, the execution of heuristic methods is not deterministic, and, on the other hand, the measured time depends on the computing system used. Moreover, it should be mentioned that the approaches presented by Farhadmanesh and Ahmadi [2020] (see Section 1) show even higher convergence rates.

But by combining the BO approach with DTW as the error function and a CWE simulation as the basis for the cutting force calculation, two additional advantages for the coefficient identification have been achieved:

- The coefficient identification can be performed without prior synchronization of measured and simulated cutting forces.

- $\quad$ The input data set may contain arbitrary CWE ratios. Changes within the identification data set are also acceptable, since the simulated GPFs contain these variations (see Section 2) as well.

In summary, the introduced requirements for the CFC determination (see Section 1) - online capability and flexible identification - are fulfilled by the described method. Up to now, the instantaneous coefficient identification is only suitable for the determination of the cutting constants $\left(K_{t c}, K_{r c}, K_{a c}\right)$ and the edge force coefficients $\left(K_{t e}, K_{r e}, K_{a e}\right)$. Runout parameters and material inhomogeneities, as introduced by Wimmer [2018] and Ehmann [1997], are currently not taken into account. This would require minor modifications of the cutting force simulation and of the optimization problem. However, the basic structure of the procedure remains unaffected. In addition, the dynamics of the dynamometer were neglected in the identification process. This is only valid if the tooth passing frequency is well below the first natural frequency of the dynamometer, which can be confirmed for the executed experiments. In high-speed machining applications, the influence of the dynamometer dynamics must either be compensated in the test data or the cutting force model must be modified to include the additional dynamics in the system. Furthermore, within the scope of this publication, directly measured cutting forces were used. For an industrial application, the use of indirectly determined process forces, as presented in Schmucker [2021], would be beneficial. 


\section{ACKNOWLEDGMENTS}

This research has been sponsored by the Federal Ministry of Education and Research (Germany) through the research project "Closed Loop Manufacturing 4.0" (grant number: 01DM19010A).

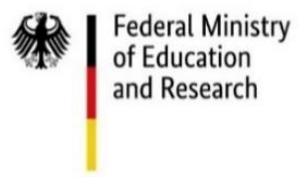

We would also like to thank the enterprise ModuleWorks for supporting this research by providing the CWE simulation API.

\section{REFERENCES}

[Albertelli 2016] Albertelli, P. et al. Model-based broadband estimation of cutting forces and tool vibration in milling through in-process indirect multiple-sensors measurements. The International Journal of Advanced Manufacturing Technology, 2016, Vol. 82, 5-8, pp. 779-796.

[Altintas 2005] Altintas, Y. et al. Virtual Machine Tool. CIRP Annals, 2005, Vol. 54, No. 2, pp. 115-138.

[Altintas and Aslan 2017] Altintas, Y. and Aslan, D. Integration of virtual and on-line machining process control and monitoring. CIRP Annals, 2017, Vol. 66, No. 1 , pp. 349-352.

[Archetti and Candelieri 2019] Archetti, F. and Candelieri, A. Bayesian Optimization and Data Science. Cham: Springer International Publishing, 2019.

[Armendia 2019] Armendia, M. et al. Twin-Control. Cham: Springer International Publishing, 2019.

[Aslan and Altintas 2018] Aslan, D. and Altintas, Y. On-line chatter detection in milling using drive motor current commands extracted from CNC. International Journal of Machine Tools and Manufacturing, 2018, Vol. 132, pp. 64-80.

[Budak 1996] Budak, E. et al. Prediction of Milling Force Coefficients From Orthogonal Cutting Data. Journal of Manufacturing Science and Engineering, 1996, Vol. 118, No. 2, pp. 216-224.

[Budak 2006] Budak, E. Analytical models for high performance milling. Part I: Cutting forces, structural deformations and tolerance integrity. International Journal of Machine Tools and Manufacture, 2006, Vol. 46, 12-13, pp. 1478-1488.

[Denkena 2012] Denkena, B. et al. Model based reconstruction of milled surface topography from measured cutting forces. International Journal of Machine Tools and Manufacture, 2012, 54-55, pp. 2533.

[Ehmann 1997] Ehmann, K.F. et al. Machining Process Modeling: A Review. Journal of Manufacturing Science and Engineering, 1997, Vol. 119, 4B, pp. 655-663.

[Farhadmanesh and Ahmadi 2020] Farhadmanesh, M. and Ahmadi, K. Online identification of mechanistic milling force models. Mechanical Systems and Signal Processing, 2020, Vol. 149.
[Gonzalo 2010] Gonzalo, O. et al. A method for the identification of the specific force coefficients for mechanistic milling simulation. International Journal of Machine Tools and Manufacture, 2010, Vol. 50, No. 9 , pp. 765-774.

[Grossi 2015] Grossi, N. et al. Speed-varying cutting force coefficient identification in milling. Precision Engineering, 2015, Vol. 42, pp. 321-334.

[Haenel 2019] Haenel, A. et al. Development of a Method to Determine Cutting Forces based on Planning and Process Data as contribution for the creation of Digital Process Twins. MM Science Journal, 2019, Vol. 2019, No. 04 , pp. 3148-3155.

[Kushner 1964] Kushner, H.J. A New Method of Locating the Maximum Point of an Arbitrary Multipeak Curve in the Presence of Noise. Journal of Basic Engineering, 1964, Vol. 86, No. 1, pp. 97-106.

[Müller 2007]. Dynamic Time Warping. In: Müller, M. Information Retrieval for Music and Motion. Berlin, Heidelberg: Springer Berlin Heidelberg. 2007, pp. 6984.

[Rivière-Lorphèvre 2017] Rivière-Lorphèvre, $E$. et al. Dynamic simulation of milling operations with small diameter milling cutters: effect of material heterogeneity on the cutting force model. Meccanica, 2017, Vol. 52, 1-2, pp. 35-44.

[Schmucker 2021] Schmucker, B. et al. Implementation of an Intelligent System Architecture for Process Monitoring of Machine Tools. Procedia CIRP, 2021, Vol. 96, No. 2, pp. 342-346.

[Scippa 2013] Scippa, A. et al. Milled Surface Generation Model for Chip Thickness Detection in Peripheral Milling. Procedia CIRP, 2013, Vol. 8, pp. 450-455.

[Stone 2014] Stone, B. Chatter and Machine Tools. Cham: Springer International Publishing, 2014.

[Wimmer 2018] Wimmer, S. et al. A cutting force model for finishing processes using helical end mills with significant runout. Production Engineering, 2018, Vol. 12 , No. 6, pp. 703-714.

[Witt 2019] Witt, M. et al. Real-time machine simulation using cutting force calculation based on a voxel material removal model. The International Journal of Advanced Manufacturing Technology, 2019, Vol. 105, 5-6, pp. 2321-2328.

[Yamada 2017] Yamada, Y. et al. Mode decoupled and sensorless cutting force monitoring based on multiencoder. The International Journal of Advanced Manufacturing Technology, 2017, Vol. 92, 9-12, pp. 4081-4093.

[Yan 2018] Yan, L. et al. Bayesian Optimization Based on K-Optimality. Entropy, 2018, Vol. 20, No. 8.

[Yesilli 2019] Yesilli, M.C. et al. Chatter Detection in Turning Using Machine Learning and Similarity Measures of Time Series via Dynamic Time Warping. [online] 2019. Available from <http://arxiv.org/pdf/1908.01678v1>.

[Zaeh 2019] Zaeh, M.F. et al. Predictive simulation of damping effects in machine tools. CIRP Annals, 2019, Vol. 68, No. 1, pp. 393-396. 